\title{
Combination therapy of conditionally replicating adenovirus and histone deacetylase inhibitors
}

\author{
DAL RAE KIM ${ }^{1}$, MI-YOUNG PARK ${ }^{1}$, HYO-JEONG LIM ${ }^{1,2}$, JONG SUN PARK ${ }^{1,2}$, YOUNG JAE CHO ${ }^{1,2}$, \\ SEI-WON LEE ${ }^{1,2}$, HO-IL YOON ${ }^{1,2}$, JAE-HO LEE ${ }^{1,2}$, YEON-SOO KIM ${ }^{3}$ and CHOON-TAEK LEE ${ }^{1,2}$ \\ ${ }^{1}$ Department of Internal Medicine, Respiratory Center, Seoul National University Bundang Hospital, Seongnam; \\ ${ }^{2}$ Division of Pulmonology and Critical Care Medicine, Lung Institute, Seoul National University, \\ College of Medicine, Seoul; ${ }^{3}$ Department of Smart Foods and Drugs and Indang \\ Insititute of Molecular Biology, Inje University, Seoul, Republic of Korea
}

Received August 31, 2011; Accepted October 7, 2011

DOI: $10.3892 /$ ijmm.2011.831

\begin{abstract}
Combination therapy of adenoviral gene therapy and a histone deacetylase (HDAC) inhibitor is important due to the enhancing effect of HDAC inhibitors on adenoviral transduction and transcription. However, contradictory results have been reported on the effect of combination of CRAd (conditionally replicating adenovirus) and HDAC inhibitors. This study was designed to investigate the interaction of CRAd and HDAC inhibitors and determine the ideal way to combine the two agents. Combination of HDAC inhibitors (SK7041, SBHA and vorinostat) at pre- and post-transductional periods with CRAd enhanced the transduction of CRAd and expression of luciferase expression from $\Delta 24$-luc in vitro. However, suppression of luciferase expression from $\Delta 24$-luc injected tumor mass was observed by in vivo tumor bioluminescence imaging and drug interaction analysis also showed an antagonistic interaction that was probably related with the inhibitory effect of the HDAC inhibitor on adenoviral replication. Suppression of $\mathrm{p} 21$ induction by $\mathrm{p} 21$ siRNA reversed the suppressive effect of vorinostat on the replication of CRAd, but still failed to reverse the antagonistic interaction. Addition of vorinostat at the pre-transductional period revealed an improvement in the transduction efficiency of CRAd and also induced a synergistic interaction between CRAd and vorinostat, which was possibly related with prevention of the suppressive effect of vorinostat on adenoviral replication. In conclusion, the addition of HDAC inhibitor before CRAd injection showed synergistic antitumor effects, which warrants further investigation on the sequence of HDAC inhibitor and CRAd treatment in an animal tumor model.
\end{abstract}

Correspondence to: Dr Choon-Taek Lee, Department of Internal Medicine, Respiratory Center, Seoul National University Bundang Hospital, 166 Gumi-Ro, Bundang-Gu, Seongnam, 463-707, Republic of Korea

E-mail: ctlee@snu.ac.kr

Key words: conditionally replication adenovirus, drug interaction, histone deacetylase inhibitor, p21

\section{Introduction}

Gene therapy for cancer has been investigated for a long time without a remarkable clinical impact. Low gene transfer rate is a major limitation for a successful gene therapy for cancer. Many experiments have reported improvements in the gene transfer rate and oncolytic virus (conditionally replicating adenovirus: CRAd) is one such example. The principle behind an oncolytic virus is the development of a viral vector that can replicate in certain conditions specific for cancer, such as p53 mutation (1), pRB/p16 inactivation $(2,3)$ and the presence of telomerase (4). Specific replication of virus in infected cancer cells induces cell lysis and the release of virus in the tumor microenvironment, after which viral vectors infect surrounding cancer cells, resulting in lateral spreading of the virus into the tumor mass.

Several steps are required for successful oncolytic viral therapy including CRAd. Highly efficient and selective transduction of CRAd into cancer cells is the first step which requires strong CAR expression on cancer cell surfaces. Highly effective transcription followed by translation of adenoviral genes is the second step, and highly efficient replication of adenovirus and subsequent cytolysis and release of adenovirus is the third step. Effective lateral spreading within the tumor is the final step for successful oncolysis (5).

Combination of HDAC inhibitors with adenovirus is another attractive strategy for cancer gene therapy. In 2001, our group reported that sodium butyrate, an HDAC inhibitor, improved antitumor effects of adenovirus-p16 by enhancing CAR expression followed by increasing the gene transfer rate (6). Many HDAC inhibitors have been reported to augment adenoviral gene therapy by enhancing CAR expression (7-10). Furthermore, HDAC inhibitors were found to enhance the transcription of adenoviral transgenes in target cells $(11,12)$. Strong synergistic interactions were found in various HDAC inhibitors and adenoviruses with the therapeutic gene $(7,8,12)$.

Encouraged by the synergistic interaction of HDAC inhibitors and adenovirus, there have been several reports on the combination of HDAC inhibitors and CRAd (13). However, contradictory results have been reported. Hoti et al reported that a HDAC inhibitor, valproic acid, inhibited the replication 
of adenovirus and consequently antagonized the cytotoxic activity of oncolytic adenovirus. They also suggested that increased p21 expression due to valproic acid was a causative mechanism of this antagonistic interaction (14). Another report showed that antitumor activities of oncolytic adenoviruses (ONYX-015, $\Delta 24)$ were enhanced by RNA interferencemediated suppression of p21 (15). It has also been reported that armed CRAd that expressed p21 shRNA showed higher stronger antitumor activity by breakdown of $\mathrm{p} 21$, the inhibitor of viral replication, due to RNA interference of p21 shRNA (16).

In contrast, several reports demonstrated that HDAC inhibitor was able to enhance the antitumor effect of oncolytic virus. Watanabe et al (17) reported that the replication-competent adenovirus (OBP-301) and the HDAC inhibitor (FR901228) showed synergistic interaction in lung cancer cell lines (A549 and NCI H460) by increasing adenovirus infectivity via up-regulation of CAR. Bieler et al (18) also reported that the triple combination of the HDAC inhibitor (trichostatin A), with chemotherapy (irinotecan) and an oncolytic adenovirus (d1520) showed synergistic interaction partly due to CAR up-regulation. Another oncolytic virus (vesicular stomatitis virus) as well as HDAC inhibitors showed synergistic interaction by dampening the innate immunity towards the oncolytic virus $(13,19)$. In another report, transfection of $\mathrm{p} 21$ promoted oncolytic adenoviral activity in ovarian cancer by increasing E1A expression (20).

Therefore, we investigated the interaction of CRAd $(\Delta 24)$ and several HDAC inhibitors in lung cancer cell lines to address these contradictory findings.

\section{Materials and methods}

Cell lines and animals. Human lung cancer cell lines (A549, NCI H460) were purchased from the American Tissue Culture Collection (Manassas, VA, USA). BALB/c nude mice were obtained from Japan SLC (Hamamatsu, Japan) and all animal experiments were approved by the Institutional Review Board (IRB) of Seoul National University Hospital.

Recombinant adenoviruses. Conditionally replicating adenoviruses $(\Delta 24)$ were provided by David T. Curiel (University of Alabama at Birmingham, USA). $\Delta 24$-luc ( $\Delta 24$ expressing luciferase) was provided by Victor van Beusechem (VU University, The Netherlands). Ad-luciferase (Ad-luc) was a CMV promoter driven, E1-deleted and replication-defective adenovirus generated in our laboratory.

HDAC inhibitors. SK7041, structurally composed of hydroxamic acid and benzamide (21), was a gift from Professor Y.J. Bang (Seoul National University Hospital). SBHA (suberoyl bishydroxamic acid) was purchased from Calbiochem (La Jolla, CA, USA). Vorinostat (suberoylanilide hydroxamic acid, SAHA) was kindly provided by Merck Sharp \& Dohme Corporation (Whitehouse Station, NJ, USA). A stock solution of vorinostat $(20 \mathrm{mM})$ was made by dissolving in DMSO and then diluting with media prior to the experiment.

Antibodies and siRNA. Antibodies to CAR, p16, pRb, p21 and actin were purchased from Santa Cruz Biotechnology
(Santa Cruz, CA, USA). p21 siRNA and control scrambled siRNA were purchased from Santa Cruz Biotechnology. p21 siRNA was transfected into lung cancer cell lines with Lipofectamine $^{\mathrm{TM}} 2000$ (Invitrogen, Carlsbad, CA, USA) according to the manufacturer's recommendations.

Effect of HDAC inhibitors on CAR expression of lung cancer cell lines. Human lung cancer cells (A549 and NCI H460) were treated with SK7041 $(0.01-1 \mu \mathrm{M})$, SBHA $(1-50 \mu \mathrm{g} / \mathrm{ml})$ or vorinostat $(0.1-10 \mu \mathrm{M})$ for $48 \mathrm{~h}$ and Western blotting for CAR was performed.

Effects of HDAC inhibitors on luciferase expression from 424-luc transduced cancer cells. Lung cancer cells (A549) were transduced with $\Delta 24$-luc (1 MOI) and HDAC inhibitors (SK7041, SBHA and vorinostat). Cells were treated with HDAC inhibitor for $12 \mathrm{~h}$ before transduction and for $48 \mathrm{~h}$ after transduction. In vitro luciferase assays were performed $48 \mathrm{~h}$ after transduction according to the manufacturer's recommendations (Luciferase assay system; Promega, Madison, WI, USA) and relative light units were measured using LMAX II $^{384}$ (Molecular Devices, Sunnyvale, CA, USA).

In vivo tumor imaging for luciferase expression. A549 lung cancer cells $\left(2 \times 10^{6}\right.$ cells/mouse) were injected into the right thighs of nude mice (female BALB/c, 6 weeks-old). Measurable tumors of 7 to $8 \mathrm{~mm}$ diameter were formed at 2 weeks. Vorinostat $(30 \mathrm{mg} / \mathrm{kg}$ ) in $100 \mu \mathrm{l}$ phosphate-buffered saline (PBS) or $100 \mu 1 \mathrm{PBS}$ were injected into the peritoneum of nude mice. The following day, Ad-luc $\left(2 \times 10^{8} \mathrm{pfu}\right)$ or $\Delta 24-\mathrm{luc}\left(2 \times 10^{8}\right.$ pfu) was injected into the tumor mass. Another treatment with vorinostat or PBS was performed $24 \mathrm{~h}$ after adenovirus injection. D-luciferin (5 mg/mouse) (Gold Biotechnology, St. Louis, MO, USA) was injected into the peritoneum, $72 \mathrm{~h}$ after adenovirus injection. In vivo bioluminescence images were captured after 10 min with the IVIS ${ }^{\text {TM }} 100$ (Caliper Life Sciences, Hopkinton, MA, USA).

Combination effects of pre- and post-transductional HDAC inhibitors on cytotoxicity of CARd. To investigate the combination effect of HDAC inhibitors on the cytotoxicity of CRAd, lung cancer cells (A549) were pretreated with HDAC inhibitors (SK7041, 100-500 nM; SBHA, 1-100 $\mu \mathrm{M}$; vorinostat, 0.1-5 $\mu \mathrm{M}$ ) for $12 \mathrm{~h}$ and transduced with $\Delta 24$ (MOI, 1-10) for $1 \mathrm{~h}$ and then treated again with HDAC inhibitors at various combinations in 96-well plates $\left(3 \times 10^{4}\right.$ cells/well). After $72 \mathrm{~h}$ of incubation, MTS [3-(4,5-dimethylthiazol-2-yl)-5-(3-carboxymethoxyphenyl)2-(4-sulfophenyl)-2H-tetrazolium] assay (CellTiter 96 Aqueous One Solution Cell Proliferation Assay; Promega, Madison, WI, USA) was performed. Interactions between CRAd and HDAC inhibitors were investigated using Calcusyn software (Biosoft, Ferguson, MO, USA) by measuring the combination index.

Analysis of cell cycle-related proteins after HDAC inhibitor treatment. Lung cancer cells (A549 and NCI H460) were treated with HDAC inhibitors (vorinostat, SBHA and SK7041) at the indicated concentrations for $48 \mathrm{~h}$. After extraction of the proteins, a Western blotting assay for pRb, p21 and p16 was performed using the SuperSignal West Pico chemiluminescent substrate kit (Thermo Scientific, Rockford, IL, USA). 
(A)

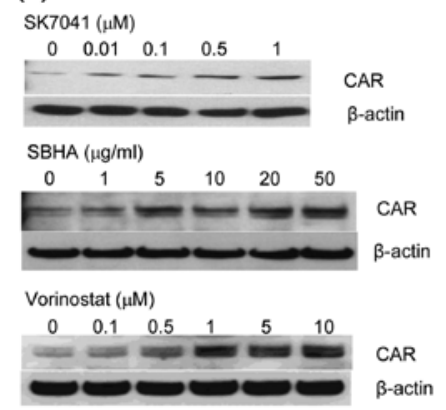

(B) RLU

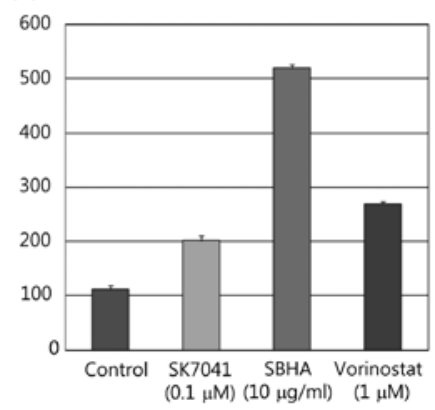

Figure 1. Effects of HDAC inhibitors on CAR and luciferase expression from $\Delta 24$-luc. (A) Western blotting assay for CAR revealed the increased expression of CAR following addition of HDAC inhibitors. Lung cancer cells (A549) were incubated with HDAC inhibitors (SK7041, SBHA and vorinostat) at indicated concentrations for $48 \mathrm{~h}$, and then a Western blotting assay for CAR was performed. (B) Increased luciferase expression from $\Delta 24$-luc transduced cells by HDAC inhibitors. Lung cancer cells were transduced with $\triangle 24$-luc (MOI, 1) and HDAC inhibitors at indicated concentrations for $12 \mathrm{~h}$ before transduction and $48 \mathrm{~h}$ after transduction. All three HDAC inhibitors increased the luciferase expression from cells transduced with $\Delta 24$-luc $(\mathrm{P}<0.05$ by ANOVA).

Suppression of the vorinostat-induced p21 expression by transfection of 21 siRNA. Lung cancer cells (A549 and NCI H460) were transfected with p21 siRNA or scrambled RNA. Cells were treated with vorinostat $(1 \mu \mathrm{M}) 6 \mathrm{~h}$ after transfection, Western blotting assay for $\mathrm{p} 21$ was performed $48 \mathrm{~h}$ after vorinostat treatment.

Change of luciferase expression from 424 -luc by p21 siRNA transfection. A549 cells were transfected with p21 siRNA (10 and $20 \mathrm{nM}$ ) for $6 \mathrm{~h}$ after which cells were transduced with $\Delta 24$-luc (MOI, 10) and vorinostat $(1 \mu \mathrm{M})$. An in vitro luciferase assay was performed after $48 \mathrm{~h}$.

Effects of HDAC inhibitors on replication of CRAd in lung cancer cells. To analyze the effect of HDAC inhibitors on the replication activity of CRAd, which is an essential characteristic of CRAd, A549 lung cancer cells ( $1 \times 10^{6}$ cells in $100-\mathrm{mm}$ plate) were transduced with $\Delta 24$ (MOI, 1) and treated with HDAC inhibitors (SK7041, $100 \mathrm{nM}$; SBHA, $10 \mu \mathrm{g} / \mathrm{ml}$; vorinostat, $1 \mu \mathrm{M})$. At $48 \mathrm{~h}$ after incubation, the adenovirus was harvested by cell lysis and concentrated by Centriplus YM-50 (Amicon, Millipore Corp. Bedford, MA, USA). Quantities of retrieving adenoviruses were calculated by measuring adenoviral concentration [tissue culture infectious dose (TCID)50 method] and lysate volume. To confirm the role of induced p 21 by vorinostat on adenoviral replication, the same experiment was repeated with vorinostat after p21 siRNA transfection to A549.

Drug interactions between $\Delta 24$ and vorinostat after p21 siRNA transfection. Lung cancer cells (A549 and NCI H460: $3 \times 10^{4}$ cells/well in 96-well plates) were transfected with p21 siRNA (20 $\mathrm{nM})$ or scrambled RNA for $6 \mathrm{~h}$ and treated with $\Delta 24$ and vorinostat (pre- and post-transductional) at various concentration combinations. At $72 \mathrm{~h}$ after transduction, cell survival was measured by the MTS assay and drug interaction was analyzed using the Calcusyn software (Biosoft, Ferguson, MO, USA).

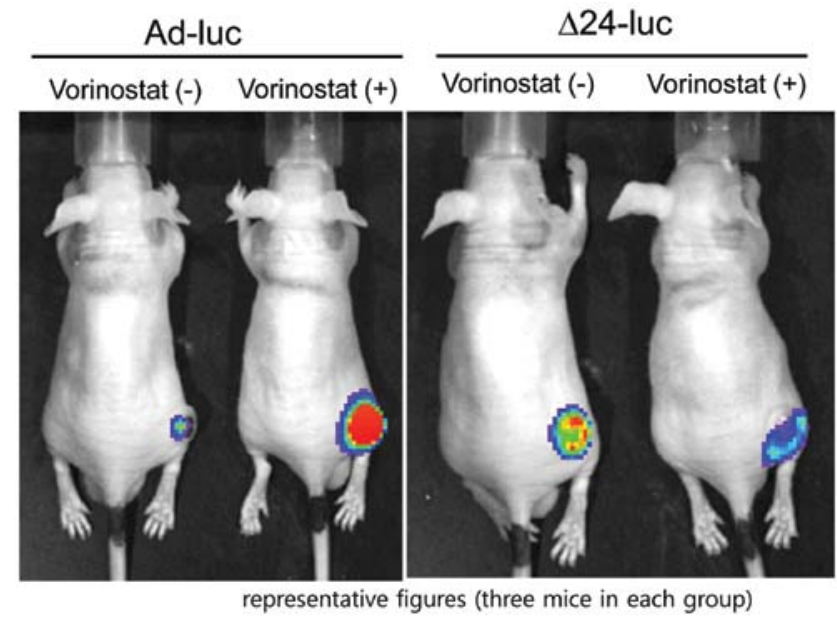

Figure 2. Effect of vorinostat on in vivo tumor imaging. Lung cancer xenograts were established by injecting A549 ( $2 \times 10^{6}$ cells/mouse) into subcutaneous tissue of nude mice (BALB/c). At 2 weeks after injection of cancer cells (day 0 ), vorinostat $(30 \mathrm{mg} / \mathrm{kg}$ in $100 \mu \mathrm{l}$ of phosphate-buffered saline) or $100 \mu \mathrm{l}$ of PBS was injected into the peritoneum on day 0 and day 2 . On day 1 , Ad-luc $\left(2 \times 10^{8} \mathrm{pfu}\right)$ or $\Delta 24$-luc $\left(2 \times 10^{8} \mathrm{pfu}\right)$ were injected intratumorally. On day 4 , D-luciferin ( $5 \mathrm{mg} /$ mouse) was injected into the peritoneum, and tumor in vivo bioluminescence imaging was captured after $10 \mathrm{~min}$. Bioluminescence for Ad-luc injected tumor increased by vorinostat, but that from the $\Delta 24$-luc injected tumor decreased.

Effect of pre- or post-transductional vorinostat treatment on luciferase expression from 424 -luc transduced cells. To differentiate the effect of vorinostat on the transduction or transcription of $\Delta 24-$ luc, lung cancer cells (A549 and NCI H460) were transduced with $\Delta 24$-luc (MOI, 1) and treated with vorinostat $(2 \mu \mathrm{M})$ for $24 \mathrm{~h}$ before (pre-transduction) or for $24 \mathrm{~h}$ after (post-transduction), or $24 \mathrm{~h}$ before and after transduction (pre- and post-transduction). The luciferase assay was performed $24 \mathrm{~h}$ after transduction.

Combination effects of pre-transductional vorinostat on cytotoxicity of CRAd. To investigate the combination effect of pre-transductional treatment of vorinostat on cytotoxicy of CRAd, lung cancer cells (A549) were treated with vorinostat (0.1-5 $\mu \mathrm{M})$ for $24 \mathrm{~h}$ and the media was removed by vigorous washing. Cells were treated with $\Delta 24$ (MOI, 1-10) for $1 \mathrm{~h}$ and complete media without vorinostat was replaced. The MTS assay was performed $96 \mathrm{~h}$ after transduction and drug interaction was analyzed.

\section{Results}

$H D A C$ inhibitors increase the expression of CAR in lung cancer cell lines. The addition of SK7041, SBHA and vorinostat increased the expression of CAR in lung cancer cell lines (A549 and NCI H460) in a dose-dependent manner (Fig. 1A).

HDAC inhibitors increase the in vitro luciferase expression from $\Delta 24$-luc. The luciferase assay from $\Delta 24$-luc transduced lung cancer cells revealed that addition of all HDAC inhibitors (pre- and post-transductional) enhanced the expression of luciferase, a transgene of $\Delta 24$-luc (Fig. 1B). This showed the potential of HDAC inhibitors for combination therapy with CRAd and HDAC inhibitors. 
(A)
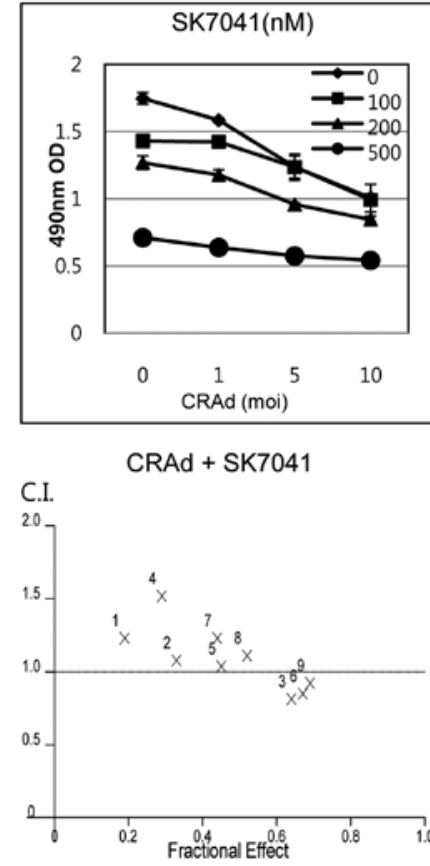

(B)

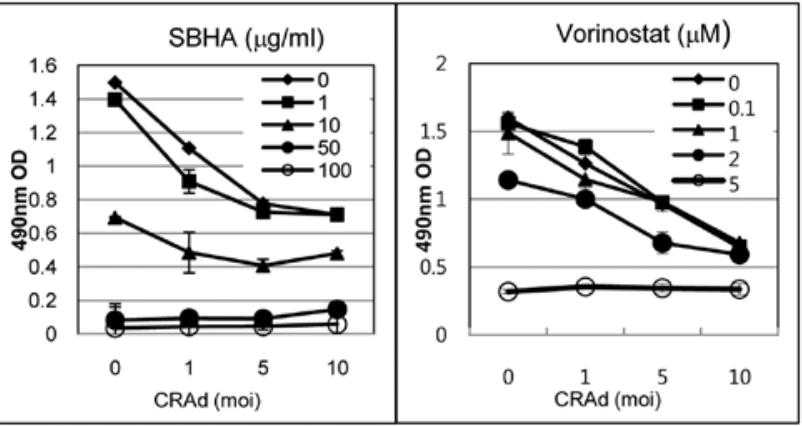

CRAd + SBHA

(C)

CRAd + vorinostat

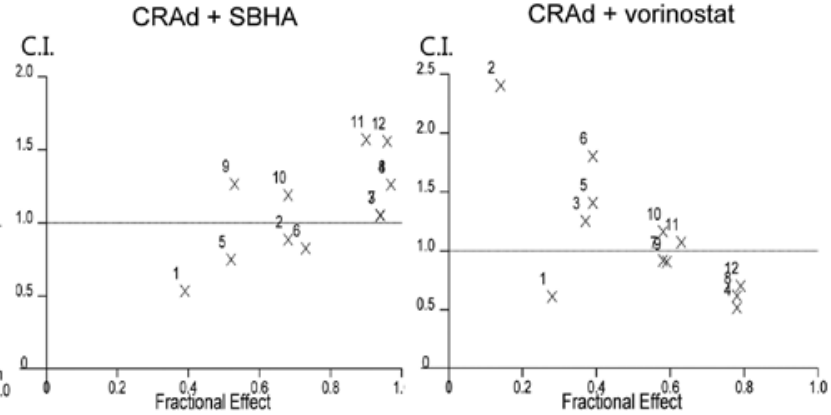

Figure 3. Antagonistic interaction of HDAC inhibitors and CRAd on lung cancer cytotoxity. A549 lung cancer cells (5x10 3 cells/well in 96-well plates) were treated with the HDAC inhibitors (A) SK7041, (B) SBHA and (C) vorinostat at the indicated concentrations pre- and post-transductional and treated with CRAd ( $\triangle 24$; MOI, 1-10). The MTS assay was performed at $72 \mathrm{~h}$ after transduction and the interactions were analyzed by the Calcusyn software. No or antagonistic interactions were found between the HDAC inhibitors and CRAd.

Suppression of in vivo luciferase expression in 424 -luc injected tumors by the HDAC inhibitor, vorinostat. Encouraged by the in vitro data, we proceeded to a similar experiment in an animal tumor model using in vivo tumor imaging for luciferase. Vorinostat increased the expression of luciferase in tumors injected with Ad-luc as was expected. However, the expression from the $\Delta 24$-luc injected tumor was suppressed by vorinostat (Fig. 2). From this contradictory finding, we speculated that a different mechanism may exist in vitro and in vivo.

Antagonistic interactions of HDAC inhibitors and CRAd on cytotoxicity in lung cancer cell lines. We analyzed the interaction of HDAC inhibitors and CRAd $(\Delta 24)$ on the cytotoxicity of lung cancer cells using the Calcusyn software. SK7041, SBHA and vorinostat showed antagonistic rather than additive or synergistic interaction in the A549 (Fig. 3) and the NCI H460 cell lines.

Effects of HDAC inhibitors on cell cycle-related proteins. HDAC inhibitors increased the expression of p21. However, absence of p16 expression in lung cancer cell lines (A549 and NCI H460) remained unchanged by HDAC inhibitors. Also, pRb levels decreased by the addition of HDAC inhibitors (Fig. 4A), and the transfection of p21 siRNA effectively suppressed p21 expression induced by vorinostat (Fig. 4B).

Increased luciferase expression by 21 siRNA transfection from lung cancer cells treated with 424 -luc and vorinostat. As showed in Fig. 1B, vorinostat $(1 \mu \mathrm{M})$ increased the expression of luciferase. Furthermore, p21 siRNA transfection (10 or $20 \mathrm{nM}$ ) induced a further increase of luciferase expression (Fig. 4C).
Decreased replication of CRAd by HDAC inhibitors reversed by 21 siRNA transfection. We calculated the replication ability of $\triangle 24$ in A549 cells in media containing HDAC inhibitors. Three HDAC inhibitors (SK7041, SBHA and vorinostat) were found to decrease the replication of $\Delta 24$ in A549 (Fig. 5A). However, suppression of p21 expression by p21 siRNA effectively reversed the replication ability of $\Delta 24$ (Fig. 5B).

Transfection of p21 siRNA could not reverse the antagonistic interaction of $\triangle 24$ and pre-and post-transductional vorinostat. Drug interaction analysis by the Calcusyn software revealed that suppression of p21 expression by $\mathrm{p} 21$ siRNA still failed to reverse the antagonistic drug interaction between $\Delta 24$ and vorinostat (Fig. 5C and D) even though p21 siRNA reversed the replication ability of $\Delta 24$.

Pre- or post-transductional vorinostat increases luciferase expression from 424-luc-transduced lung cancer cells. Pre-transduction, post-transduction and both pre- and post-transduction vorinostat increased the expression of luciferase, but the enhancement of luciferase expression was the weakest in pre-transductional vorinostat (Fig. 6A and B). This finding is consistent with our previous experiment that vorinostat showed a dual effect on adenoviral gene expression by increasing transduction through elevated CAR and by increasing transcription of the adenoviral transgene (12).

Pre-transductional vorinostat increases the cytotoxic effects of 424 on lung cancer cells. In contrast to pre- and post-transductional vorinostat (Fig. 3), pre-transductional treatment of lung cancer with vorinostat increased the cytotoxicity of CRAd $(\Delta 24)$ on lung cancer cells (Fig. 6C and D). This synergistic 
(A)

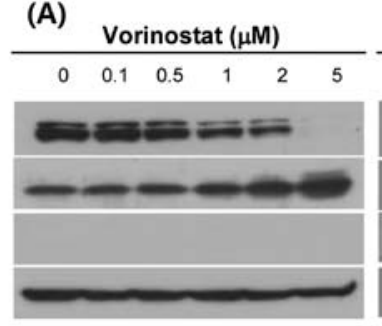

(B)

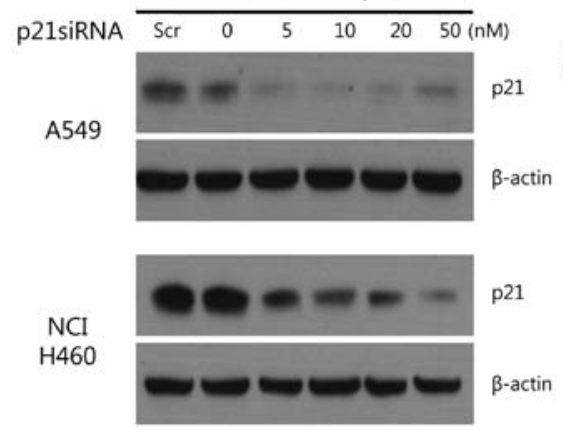

$\mathrm{SBHA}(\mu \mathrm{g} / \mathrm{ml})$
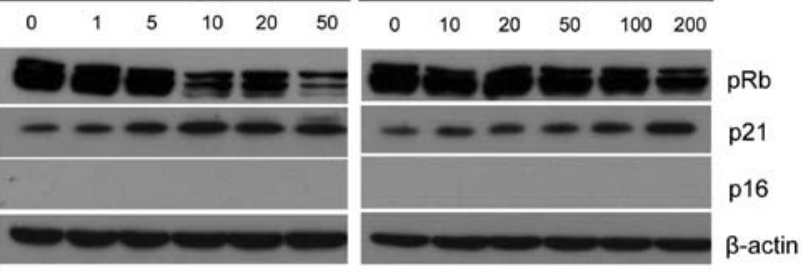

(C)

$\Delta 24-$ luc (10moi, A549)

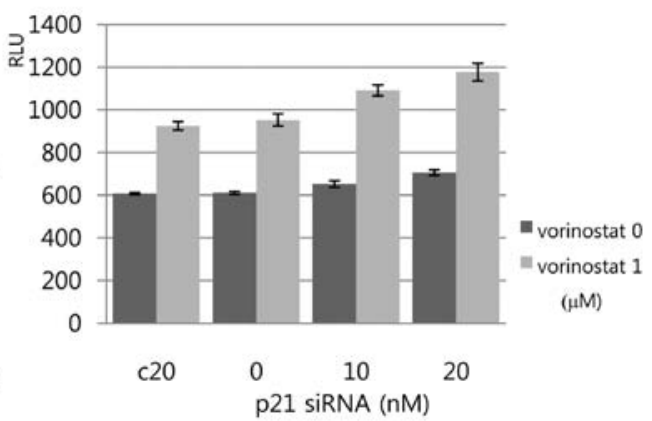

Figure 4. (A) Changes of cell cycle related proteins by HDAC inhibitors. Addition of HDAC inhibitors induced a decrease in the expression of pRb and an increase in the expression of p21 in A549 lung cancer cells. (B) Transfection of p21 siRNA effectively suppressed the p21 expresion induced by vorinostat (C) Suppression of p21 induction due to vorinostat by p21 siRNA (10,20 nM) significantly enhanced the increase of luciferase by vorinostat compared with scrambled siRNA (c20) and no siRNA ( $\mathrm{P}<0.05$ by ANOVA).

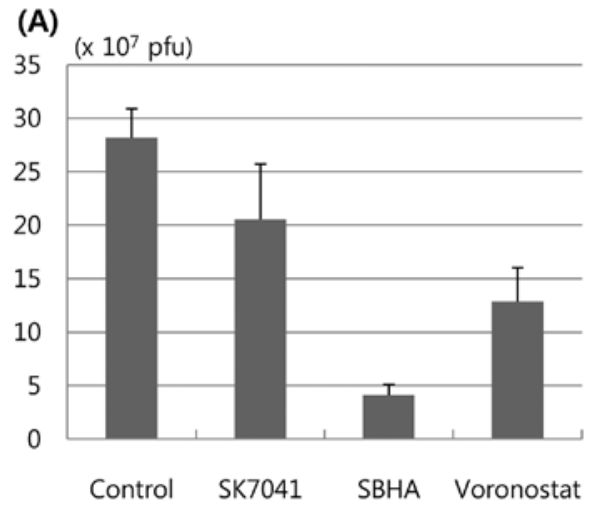

(B) $\left(\times 10^{7} \mathrm{pfu}\right)$

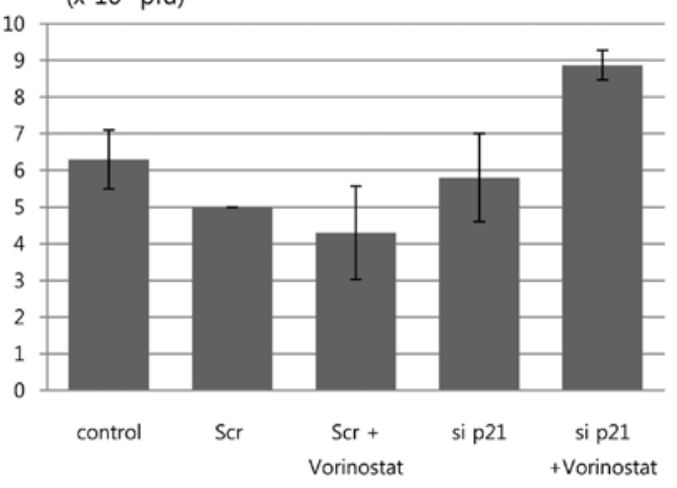

(C) CRAd + vorinostat (si P21) - Algebraic estimate

(D) CRAd + vorinostat (Scr) - Algebraic estimate
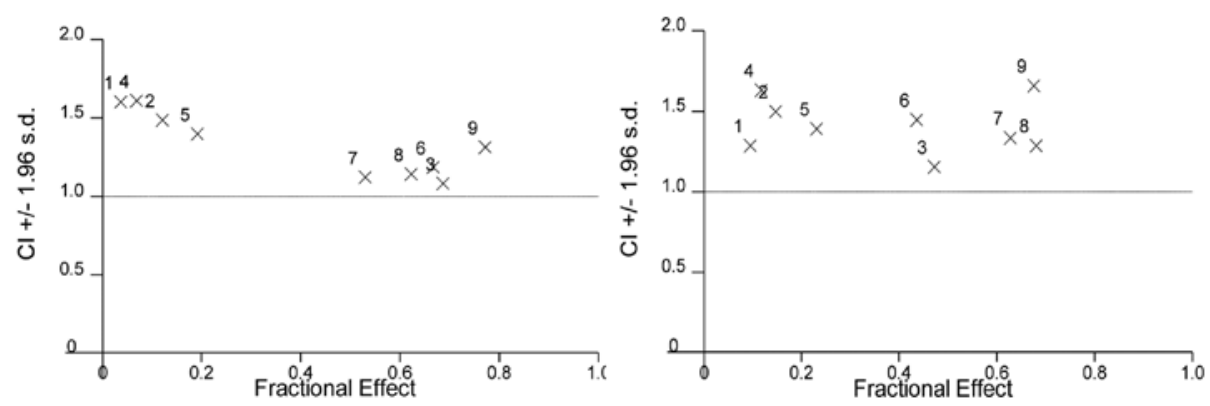

Figure 5. (A) Suppression of CRAd replication by HDAC inhibitors. Lung cancer cells (A549) were co-treated with CRAd ( $224: 1$ MOI) and HDAC inhibitors (SK7041, $100 \mathrm{nM}$; SBHA, $10 \mu \mathrm{g} / \mathrm{ml}$; vorinostat, $1 \mu \mathrm{M}$ ). Adenoviruses were harvested from cell lysate and concentrated with Centriprep 30 after $48 \mathrm{~h}$. Retrieved adenoviruses were calculated by measuring adenoviral concentration by the TCID50 method. All three HDAC inhibitors significantly reduced replication of CRAd ( $\mathrm{P}<0.05$ by ANOVA). (B) Recovery of CRAd replication by pretreatment with $\mathrm{p} 21$ siRNA. To confirm the role of vorinostat-induced 21 on adenoviral replication, the same experiment was repeated with vorinostat after p21 siRNA transfection to A549. Suppression of p21 by p21 siRNA effectively reversed the suppression of CRAd's replication. The retrieved amount of CRAd after p21 siRNA and vorinostat was significantly increased compared with that of untreated control, scrambled siRNA control and p21 siRNA alone $(\mathrm{P}<0.05)$. (C and D) Interaction of vorinostat (pre- and post-transductional) and CRAd after p21 siRNA transfection. Lung cancer cells were transfected with (C) p21 siRNA (20 nM) or (D) scrambled siRNA. At $24 \mathrm{~h}$, vorinostat at indicated concentrations were treated for $6 \mathrm{~h}$ followed by transduction with CRAd at indicated MOI for $1 \mathrm{~h}$ and then treated with vorinostat. At $72 \mathrm{~h}$ after transduction, cell survival was measured by the MTS assay and drug interaction was analyzed. Antagonistic interaction of vorinostat and CRAd was still observed. 
(A)

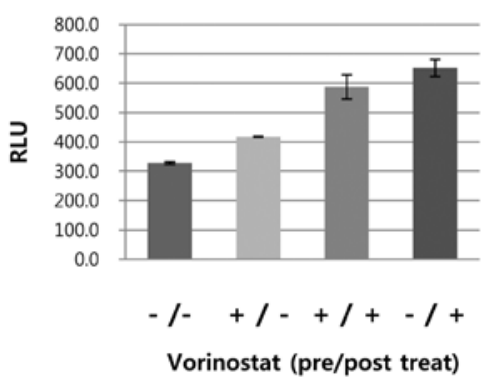

(C)

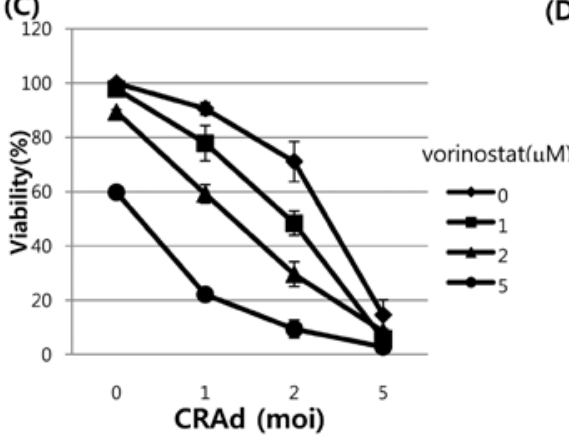

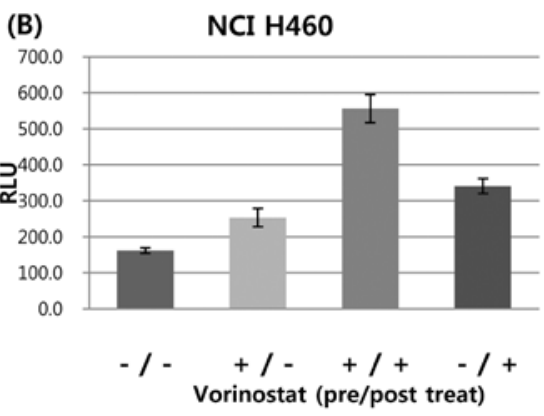

(D)

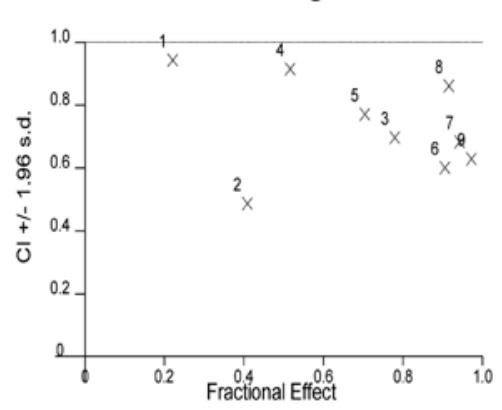

Figure 6. (A and B) Effect of pre-transductional or post-transductional vorinostat treatment on luciferase expression from $\Delta 24$-luc transduced cells. Cancer cells were treated with vorinostat before, after or before and after transduction with $\Delta 24$-luc to A549. Pre-transductional vorinostat increased luciferase expression compared with untreated control. However, pre-and post- or post-transductional vorinostat showed stronger luciferase expression than pre-transductional vorinostat. (C and D) Synergistic interaction of CRAd and pre-transductional vorinostat. The same experiment for drug interaction analysis was performed with $\Delta 24$ and pre-transductional vorinostat. Most of combination indices analyzed by Calcusyn software were between $0.5-0.9$ suggesting synergistic interaction between $\Delta 24$ and pre-transductional vorinostat.

effect may be related to the increased transduction of $\Delta 24$ mediated by increased CAR expression induced by vorinostat.

\section{Discussion}

Contradictory results have been reported on the combination effect of CRAd and HDAC inhibitors (13). HDAC inhibitors are widely investigated for combination therapy with adenoviral gene therapy since they can increase the expression of CAR, which is a major cellular receptor for adenovirus entry on the cell surface, resulting in the enhanced transduction of the adenovirus. Therefore, it is logical to investigate the combination therapy of CRAd and HDAC inhibitors. Effective transduction into target cells, high transcription and expression of viral proteins, as well as highly efficient viral replication in cells are essential for the successful antitumor effects of CRAd (5).

We found that the addition of HDAC inhibitors (SK7041, SBHA and vorinostat) increased CAR expression in the lung cancer cell lines tested. Furthermore, HDAC inhibitors also increased luciferase expression from $\Delta 24$-luc (CRAd carrying luciferase reporter gene). These findings strongly suggest that HDAC inhibitors increased the transduction efficiency of CRAd and the transcription of the transgene of CRAd.

However, intraperitoneal injection of vorinostat clearly decreased the expression of luciferase from $\Delta 24$-luc injected tumor mass in animal tumor models and combination therapy of HDAC inhibitor and CRAd in lung cancer cell lines showed an antagonistic interaction.

How can we explain these contradictory results? In the in vitro experiment, transduction and transcription of $\Delta 24$-luc were more important for luciferase expression compared to the replication of $\Delta 24$ in tumor cells. However, replication and lateral spread of CRAd was more important for luciferase expression than $\Delta 24$-luc injected tumor in the in vivo tumor model and the in vitro cytotoxicity assay.

As reported already (14), increased p21 expression due to HDAC inhibitors seems to be a causative mechanism since the addition of HDAC inhibitors to CRAd-transduced lung cancer cells reduced the numbers of CRAd from cells while silencing of p21 expression by p21 siRNA increased the replication of CRAd. Furthermore, p21 siRNA treatment as well as vorinostat also increased luciferase expression and replication of CRAd.

Therefore, we confirmed that concurrent treatment of HDAC inhibitors and CRAd induced antagonistic interactions due to reduction of CRAd replication, even though HDAC inhibitors increased adenoviral transduction and transcription.

How can we solve this discrepancy? For successful oncolysis by CRAd, high transduction and transcription rate and efficient replication are essential. Suppression of p21 induction by HDAC inhibitors using RNA interference technique could be an attractive strategy. Shiina et al (15) suggested that the cytopathic effect of CRAd (ONYX-015, $\Delta 24)$ and the production of adenoviruses were enhanced by siRNA-mediated knockdown of p21. Oncolytic adenovirus expressing therapeutic genes (armed therapeutic oncolytic adenovirus) could be a realistic solution. Oncolytic adenovirus expressing siRNA may induce the synergy between oncolysis due to CRAd, and the antitumor effect due to siRNA (22). A CRAd expressing p21 shRNA seems to be a reasonable alternative to suppress p21 (16). It was demonstrated that CRAd carrying p21 shRNA showed an increase in viral replication and viral oncolysis of 
prostate cancer cells and prevention of tumor growth by intratumoral injection. However, p21 siRNA transfection failed to reverse the antagonistic drug interaction between CRAd and vorinostat. Furthermore, applying p21 siRNA in a clinical setting is not a realistic option.

According to our current results and previous reports on CRAd and HDAC inhibitors, it is reasonable to summarize that HDAC inhibitors increased the transduction of CRAd by enhanced CAR expression and increased the transgene expression of CRAd by stimulation of the transcription of the viral transgene. However, HDAC inhibitors inhibited the replication of CRAd following p21 induction.

Is there any other strategy to overcome this dilemma? A pharmacokinetic study of vorinostat revealed that the $t^{1} / 2$ (halflife) of vorinostat was $1.8 \mathrm{~h}$ after single injection and most of vorinostat was cleared $8 \mathrm{~h}$ after injection in humans (23).

We hypothesized that exposure of vorinostat only before transduction of CRAd could improve the antitumor effect of CRAd by increasing transduction and transcription and by avoiding the inhibitory effect on viral replication. We analyzed the enhancing effect of vorinostat on $\Delta 24$-luc expression according to the timing and duration of vorinostat treatment. Both pre- and post-transductional vorinostat increased luciferase expression, however, the enhancing effect of posttransductional vorinostat on $\Delta 24$-luc was more potent than that of pre-transductional vorinostat. This finding was consistent with our previous findings showing the effects of vorinostat on Ad-luc (replication incompetent adenovirus). After confirmation that pre-transductional vorinostat increased luciferase expression, we investigated the combination antitumor effects of $\Delta 24$, as well as pre-transductional vorinostat. In contrast to antagonistic interaction of $\Delta 24$ and pre- and post-transductional vorinostat, pre-transductional vorinostat induced additive to synergistic interaction.

From these observations, we concluded that administration of vorinostat before CRAd injection may improve the antitumor effect by increasing transduction of CRAd and avoiding the inhibitory effect of vorinostat on adenoviral replication. We are planning further experiments in an animal tumor model to determine the appropriate timing of vorinostat and CRAd administration for maximal antitumor effects.

\section{Acknowledgements}

This study was supported by a grant from the National Research Foundation of Korea (2011-0002169) and a grant from the Seoul National University Bundang Hospital (03-2005-001) to C.-T. Lee.

\section{References}

1. Heise C, Sampson-Johannes A, Williams A, McCormick F, Von Hoff DD and Kirn DH: ONYX-015, an E1B gene-attenuated adenovirus, causes tumor-specific cytolysis and antitumoral efficacy that can be augmented by standard chemotherapeutic agents. Nat Med 3: 639-645, 1997.

2. Heise C, Hermiston T, Johnson L, et al: An adenovirus E1A mutant that demonstrates potent and selective systemic antitumoral efficacy. Nat Med 6: 1134-1139, 2000.
3. Fueyo J, Gomez-Manzano C, Alemany R, et al: A mutant oncolytic adenovirus targeting the $\mathrm{Rb}$ pathway produces antiglioma effect in vivo. Oncogene 19: 2-12, 2000.

4. Huang TG, Savontaus MJ, Shinozaki K, Sauter BV and Woo SL: Telomerase-dependent oncoly tic adenovirus for cancer treatment. Gene Ther 10: 1241-1247, 2003.

5. Yamamoto M and Curiel DT: Current issues and future directions of oncolytic adenoviruses. Mol Ther 18: 243-250, 2010.

6. Lee CT, Seol JY, Park KH, et al: Differential effects of adenovirus-p16 on bladder cancer cell lines can be overcome by the addition of butyrate. Clin Cancer Res 7: 210-214, 2001.

7. Fan S, Maguire CA, Ramirez SH, et al: Valproic acid enhances gene expression from viral gene transfer vectors. J Virol Methods 125: 23-33, 2005.

8. Goldsmith ME, Kitazono M, Fok P, Aikou T, Bates S and Fojo T: The histone deacetylase inhibitor FK228 preferentially enhances adenovirus transgene expression in malignant cells. Clin Cancer Res 9: 5394-5401, 2003.

9. Kitazono M, Goldsmith ME, Aikou T, Bates S and Fojo T: Enhanced adenovirus transgene expression in malignant cells treated with the histone deacetylase inhibitor FR901228. Cancer Res 61: 6328-6330, 2001.

10. VanOosten RL, Earel JK Jr, and Griffith TS: Histone deacetylase inhibitors enhance Ad5-TRAIL killing of TRAIL-resistant prostate tumor cells through increased caspase-2 activity. Apoptosis 12: 561-571, 2007.

11. Dion LD, Goldsmith KT, Tang DC, Engler JA, Yoshida M and Garver RI Jr: Amplification of recombinant adenoviral transgene products occurs by inhibition of histone deacetylase. Virology 231: 201-209, 1997.

12. Kim DR, Park MY, Lee CS, et al: Combination of vorinostat and adenovirus-TRAIL exhibits a synergistic antitumor effect by increasing transduction and transcription of TRAIL in lung cancer cells. Cancer Gene Ther 18: 467-477, 2011.

13. Nguyen TL, Wilson MG and Hiscott J: Oncolytic viruses and histone deacetylase inhibitors - a multi-pronged strategy to target tumor cells. Cytokine Growth Factor Rev 21: 153-159, 2010.

14. Hoti N, Chowdhury W, Hsieh JT, Sachs MD, Lupold SE and Rodriguez R: Valproic acid, a histone deacetylase inhibitor, is an antagonist for oncolytic adenoviral gene therapy. Mol Ther 14: 768-778, 2006.

15. Shiina M, Lacher MD, Christian C and Korn WM: RNA interference-mediated knockdown of p21(WAF1) enhances anti-tumor cell activity of oncolytic adenoviruses. Cancer Gene Ther 16: 810-819, 2009

16. Hoti N, Chowdhury WH, Mustafa S, et al: Armoring CRAds with p21/Waf-1 shRNAs: the next generation of oncolytic adenoviruses. Cancer Gene Ther 17: 585-597, 2010.

17. Watanabe T, Hioki M, Fujiwara T, et al: Histone deacetylase inhibitor FR901228 enhances the antitumor effect of telomerasespecific replication-selective adenoviral agent OBP-301 in human lung cancer cells. Exp Cell Res 312: 256-265, 2006.

18. Bieler A, Mantwill K, Dravits T, et al: Novel three-pronged strategy to enhance cancer cell killing in glioblastoma cell lines: histone deacetylase inhibitor, chemotherapy, and oncolytic adenovirus d1520. Hum Gene Ther 17: 55-70, 2006.

19. Nguyen TL, Abdelbary H, Arguello M, et al: Chemical targeting of the innate antiviral response by histone deacetylase inhibitors renders refractory cancers sensitive to viral oncolysis. Proc Natl Acad Sci USA 105: 14981-14986, 2008.

20. Flak MB, Connell CM, Chelala C, et al: p21 Promotes oncolytic adenoviral activity in ovarian cancer and is a potential biomarker. Mol Cancer 9: 175, 2010.

21. Kim IA, Shin JH, Kim IH, et al: Histone deacetylase inhibitormediated radiosensitization of human cancer cells: class differences and the potential influence of p53. Clin Cancer Res 12: 940-949, 2006

22. Pei DS, Di JH, Chen FF and Zheng JN: Oncolytic-adenovirusexpressed RNA interference for cancer therapy. Expert Opin Biol Ther 10: 1331-1341, 2010.

23. Ramalingam SS, Parise RA, Ramanathan RK, et al: Phase I and pharmacokinetic study of vorinostat, a histone deacetylase inhibitor, in combination with carboplatin and paclitaxel for advanced solid malignancies. Clin Cancer Res 13: 3605-3610, 2007. 